Check for updates

Cite this: RSC Adv., 2019, 9, 30575

\title{
Synthesis of porous carbon via a waste tire leavening strategy for adsorptive desulfurization
}

\author{
Yanhong Chao, (D) a Haitao Ju, ${ }^{\mathrm{b}}$ Jing Luo, ${ }^{\mathrm{C}}$ Yan Jin, ${ }^{\mathrm{a}}$ Chao Wang, ${ }^{\mathrm{c}}$ Jun Xiong, ${ }^{\mathrm{c}}$ \\ Peiwen $\mathrm{Wu}^{,}{ }^{\mathrm{c}}$ Haiyan $\mathrm{Ji}{ }^{* \mathrm{~b}}$ and Wenshuai Zhu (D) *c
}

Adsorptive desulfurization is an effective technology for removing harmful sulfur under mild conditions. Carbon materials have many advantages and are often used in adsorption desulfurization research, but until now have been synthesized using complicated methods and have shown limited adsorption capacity. Using an $\mathrm{NaHCO}_{3}$-assisted leavening method, waste tires were in the current work used as raw materials to produce hierarchically porous carbon that exhibits a high specific surface area and abundant oxygen-containing functional groups. In contrast to the sulfur removal by the carbon material prepared using a commonly used method, the as-prepared carbon material shows excellent adsorption performance, and was able to achieve an ultra-deep desulfurization of pentanethiol, specifically removing up to $99.7 \%$ of the sulfur from a model fuel with an initial sulfur concentration of $28 \mathrm{ppm}$. Therefore, we have provided a simple method for synthesizing adsorbents with high adsorption performance, and we expect these adsorbents to be used for industrial adsorptive desulfurization.

Received 9th August 2019

Accepted 11th September 2019

DOI: 10.1039/c9ra06195a

rsc.li/rsc-advances graphene, ${ }^{21}$ and boron nitride, ${ }^{32}$ among others. Of these different types of adsorbents, activated carbon has shown some particularly excellent properties such as high adsorption capacity, ${ }^{33,34}$ high specific surface area ${ }^{35,36}$ and easy regeneration. The raw materials that have been recently reported to be used for the synthesis of activated carbon have included sewage sludge, biochar, waste rubber tires, wood, lignocellulosic biomass, and animal bones. ${ }^{37}$ Rubber tires are essential for our daily transportation, and since they readily wear out, many waste tires become available every year all over the world. However, waste tires are non-biodegradable materials, ${ }^{37}$ and hence pose environmental problems. These problems, however, could be solved by recycling and reusing the tires. A previously reported method for preparing activated carbon materials from waste tires ${ }^{37-44}$ involves carbonizing the raw materials at about $500{ }^{\circ} \mathrm{C}$, and subjecting the resulting sample to a physical activation process and then an $\mathrm{HNO}_{3}$ and $\mathrm{NaOH}$ chemical activation (Table 1). In addition, metals and metal oxides have been added to the activated carbon material to further enhance the adsorption performance. ${ }^{39,42}$ Therefore, in order to avoid the introduction of metals and to simplify the synthesis step, further investigations of the synthesis method have become extremely important. The "leavening" method has been reported to be advantageous for increasing the specific surface area of carbon materials. ${ }^{45,46}$

Herein, to develop a green and sustainable synthetic process starting with waste tires as raw materials, we deployed the leavening method and obtained carbon materials displaying high specific surface areas and abundant surface functional groups. The obtained adsorbent was investigated in the
${ }^{b}$ School of Materials Science and Engineering, Jiangsu University, Zhenjiang 212013, P. R. China.E-mail: hyji1013@ujs.edu.cn

${ }^{c}$ School of Chemistry and Chemical Engineering, Jiangsu University, Zhenjiang 212013, P. R. China.E-mail: zhuws@ujs.edu.cn 
Table 1 The adsorption performance of activated carbon materials from waste tires

\begin{tabular}{|c|c|c|c|c|}
\hline Entry & Adsorbent & Initial concentration & $\begin{array}{l}\text { Adsorption capacity } \\
\text { (mg S per g adsorbent) or } \\
\text { sulfur removal }\end{array}$ & Ref. \\
\hline 1 & $\begin{array}{l}\text { Acidic and basic treatments } \\
\text { of activated } \mathrm{C}\end{array}$ & $\begin{array}{l}\text { T (51 ppm), BT (50 ppm) DBT } \\
(52 \mathrm{ppm})\end{array}$ & $0.18(\mathrm{~T}), 0.50(\mathrm{BT}), 1.20(\mathrm{DBT})$ & 38 \\
\hline 2 & $\begin{array}{l}\text { Ce/Fe-loaded rubber tire- } \\
\text { derived activated C }\end{array}$ & $\begin{array}{l}\text { T (52 ppm), BT (50 ppm) DBT } \\
(51 \mathrm{ppm})\end{array}$ & 7.78(T), 7.23(BT), 16.63(DBT) & 37 \\
\hline 3 & Rubber tire-derived C & DBT (50 ppm) & 8.60 & 40 \\
\hline 4 & $\begin{array}{l}\text { Nickel/rubber tire-derived } \\
\text { activated C }\end{array}$ & DBT (59 ppm) & $96.00 \%$ & 39 \\
\hline 5 & $\begin{array}{l}\text { Nanocomposite of rubber } \\
\text { tire-derived activated } \mathrm{C} \text { and } \\
\text { manganese oxide }\end{array}$ & $\begin{array}{l}\text { T (50 ppm), BT (52 ppm) DBT } \\
(53 \mathrm{ppm})\end{array}$ & $4.50(\mathrm{~T}), 5.70(\mathrm{BT}), 11.40(\mathrm{DBT})$ & 41 \\
\hline 6 & $\begin{array}{l}\text { Cobalt and molybdenum } \\
\text { nanoparticles loaded on } \\
\text { rubber tire-derived } \\
\text { activated C }\end{array}$ & $50 \mathrm{ppm}$ each & $\begin{array}{l}2.20(\mathrm{~T}), 2.32(\mathrm{BT}), 2.72(\mathrm{DBT}) \\
\text { 3.68(MBT), 3.64(MDBT), } \\
\text { 3.72(DMDBT) }\end{array}$ & 42 \\
\hline 7 & Waste tires to activated $\mathrm{C}$ & DBT (150 ppm) & 1.22 & 43 \\
\hline 8 & $\begin{array}{l}\text { Porous } \mathrm{C} \text { from waste tire } \\
\text { leavening }\end{array}$ & Pentanethiol (30 ppm S) & $99.70 \%$ & This work \\
\hline
\end{tabular}

desulfurization of pentanethiol and butanethiol. As expected, the carbon material prepared using the leavening method demonstrated a much enhanced adsorptive desulfurization ability compared to general carbon materials. This result suggests the significance here of randomly opened macropores, high specific surface area and abundant oxygen-containing functional groups of the as-prepared carbon materials. Moreover, this carbon material when optimized achieves very extensive desulfurization at a low concentration of sulfur, indicating promising application prospects for this material.

\section{Experimental}

\subsection{Materials}

KOH (AR grade) was purchased from Chengdu Kelong Chemical Reagent Factory. $\mathrm{NaHCO}_{3}$ (AR grade) was obtained from Sinopharm Chemical Reagent Co. Pentanethiol (>99.5\%) was purchased from TCI (Shanghai) Development Co., Ltd. and butanethiol (>99\%) was purchased from Aladdin.

\subsection{Preparation of the carbon materials}

First, $5.05 \mathrm{~g}$ of waste rubber tire particles and $10.2 \mathrm{~g}$ of $\mathrm{KOH}$ were ground and mixed, and then transferred into $50 \mathrm{~mL}$ of distilled water and stirred at $60{ }^{\circ} \mathrm{C}$. The purpose of this process was to remove the surface impurities of the waste tires. The obtained sample was filtered, washed and dried. Then, the resulting black solid was mixed with $\mathrm{NaHCO}_{3}$ in mass ratios of $1: 1,1: 1.5$ and $1: 2$, respectively. Finally, the obtained mixture was calcined at $800{ }^{\circ} \mathrm{C}$ for $2 \mathrm{~h}$ under a nitrogen atmosphere. The prepared materials were marked as C-1, C-1.5 and C-2 (Fig. 1a).

Similar to the above process, the carbon material was also pre-treated and then calcined, but the calcination process was carried out without $\mathrm{NaHCO}_{3}$ and marked as C.

\subsection{Characterization of the materials}

Scanning electron microscopy (SEM) measurements were carried out using a JSM-6010 PLUS/LA scanning electron microscope. Surface areas of the obtained materials were determined using the Brunauer-Emmett-Teller (BET) method based on $\mathrm{N}_{2}$ adsorption-desorption isotherms collected on a TriStar II 2020 surface area and porosity analyser (Micromeritics Instrument Corporation). Fourier-transform infrared (FT-IR) spectra were acquired using a Nicolet FTIR spectrophotometer (Nexus 470). Raman spectra (Thermo Scientific DXR) were acquired to further characterize the properties of the materials.

\subsection{Adsorption experiments}

Model fuels with different initial sulfur concentrations $(15,28$, 38 , and $48 \mathrm{ppm}$ ) were each prepared by dissolving an
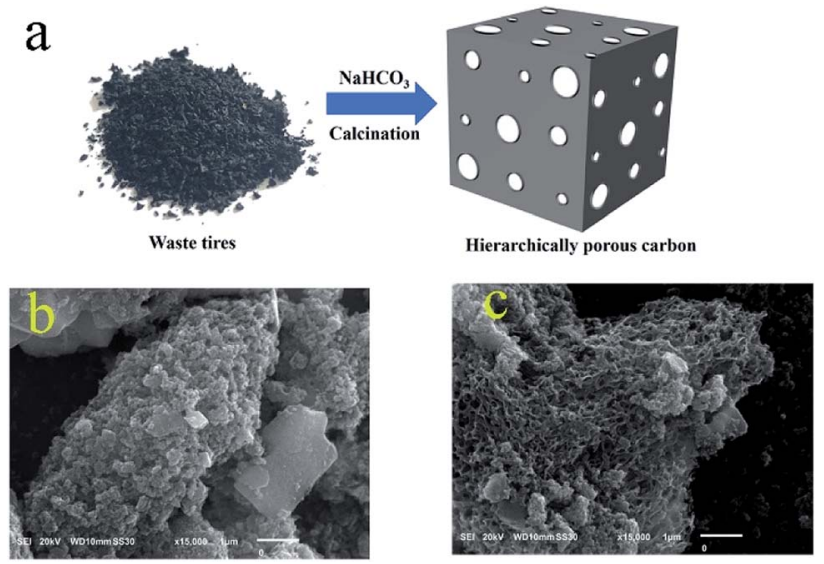

Fig. 1 (a) Schematic of the synthesis of porous carbon. (b and c) SEM images of $\mathrm{C}$ and $\mathrm{C}-1.5$. 
appropriate amount of pentanethiol in n-octane. A similar method was used to prepare a butanethiol model fuel containing 27 ppm S. The adsorption process involved adding $0.1 \mathrm{~g}$ of adsorbent and $20 \mathrm{~mL}$ of model fuel to a $50 \mathrm{~mL}$ conical flask and stirring this mixture at a certain temperature. A gas chromatography-flame photometric detector was used to determine the residual sulfur concentration.

The equilibrium adsorption capacity $\left(q_{\mathrm{e}}\right)$ values of the sulfur compounds were determined using the equation

$$
q_{\mathrm{e}}=\left(C_{0}-C_{\mathrm{e}}\right) V / m
$$

The equation

$$
\text { Sulfur removal }(\%)=\left(1-C_{t} / C_{0}\right) \times 100 \%
$$

was also used. In these equations, $q_{\mathrm{e}}, C_{0}, C_{t}, C_{\mathrm{e}}, V$ and $m$ represent the equilibrium adsorption capacity $(\mathrm{mg} S$ per $\mathrm{g}$ adsorbent), initial sulfur content (ppm), sulfur content (ppm) at $t$ min, equilibrium sulfur content (ppm), volume of the model fuel (L) and mass of the adsorbent (g), respectively.

\section{Results and discussion}

\subsection{Characterization of the carbon materials}

The morphologies of the as-prepared carbon materials were investigated using a scanning electron microscope (SEM). ${ }^{\text {47-50 }}$ As shown in Fig. 1b, the bulk carbon material obtained by directly calcining waste tires was observed in SEM images to display a rough surface. When $\mathrm{NaHCO}_{3}$ was mixed with the waste tire and calcined, the obtained carbon material showed many randomly open macropores (Fig. 1c). The presence of macropores was beneficial to increase the specific surface area of the carbon material and expose more interior atoms. These properties contributed to the exposure of the adsorption sites and hence to the promotion of the adsorption performance; ${ }^{51}$ moreover, the macropores facilitated mass transfer, ${ }^{52}$ that is, the adsorbents could easily contact the sulfides. Therefore, the carbon material prepared by the "leavening" method may be more favorable for adsorptive desulfurization.

The specific surface areas and pore size distributions of the carbon materials were characterized by acquiring their $\mathrm{N}_{2}$ adsorption-desorption isotherms (Fig. 2). ${ }^{53}$ These isotherms each showed a typical II type curve with an H3 hysteresis loop,
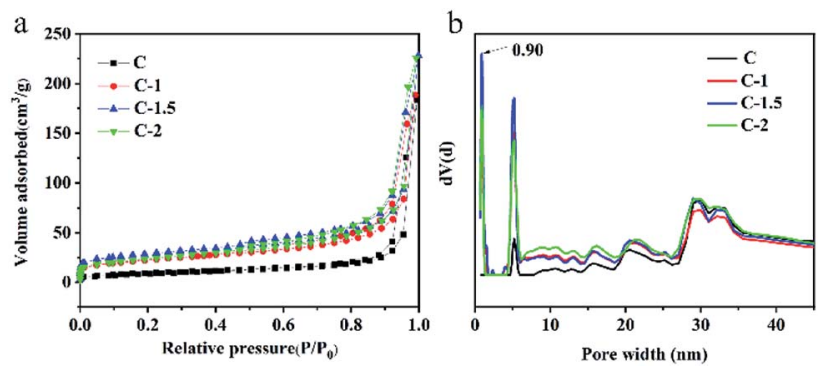

Fig. 2 (a) $\mathrm{N}_{2}$ adsorption-desorption isotherms and (b) the pore size distribution of C, C-1, C-1.5, and C-2. indicating the presence of micropores and mesopores in the carbon material. Based on the BET method and quenched solidstate functional theory (QSDFT), the specific surface areas and pore size distributions of the carbon materials were obtained. The specific surface area of C-1.5 $\left(99.3 \mathrm{~m}^{2} \mathrm{~g}^{-1}\right)$ was about three times that of $\mathrm{C}\left(32.8 \mathrm{~m}^{2} \mathrm{~g}^{-1}\right)$. This case was distinguished by the addition of $\mathrm{NaHCO}_{3}$ having generated a large amount of gas $\left(\mathrm{CO}_{2}\right)$ during the calcination process and the activator having reacted with carbon intermediates, ${ }^{46}$ resulting in the production of carbon material containing many pores and surface functional groups. The characterization result was consistent with the above-described SEM results. Moreover, according to the literature, a high specific surface area is beneficial for enhancing adsorption performance and macropores and mesopores contribute to mass transfer, ${ }^{54}$ so the carbon material prepared using the leavening method was expected to have the potential to display excellent adsorption performance.

To gain further insight into the surface chemical functional groups of the carbon materials, FT-IR spectra were acquired. ${ }^{55-58}$ As shown in Fig. 3, bands corresponding to a C$\mathrm{OH}$ stretching mode and bending mode were found at 3417 and $1620 \mathrm{~cm}^{-1} .{ }^{59}$ Additionally, a band was observed at about $1031 \mathrm{~cm}^{-1}$ and was attributed to the infrared vibration of the $\mathrm{C}-\mathrm{O}$ bond. ${ }^{60} \mathrm{~A}$ band was also observed at about $1428 \mathrm{~cm}^{-1}$ and may have been due to the asymmetric bending vibration of $\mathrm{C}-$ $\mathrm{H}^{57}$ These results illustrate that many oxygen atoms are incorporated in the carbon materials prepared using the leavening method.

Raman spectroscopy is the most direct and nondestructive technique used to characterize the structure of carbon materials. Usually, it is employed to characterize the defects, disorder and doping of carbon materials. As shown in Fig. 4, D and $\mathrm{G}$ bands are located at about 1340 and $1600 \mathrm{~cm}^{-1} \cdot{ }^{61}$ It is generally believed that the $\mathrm{G}$ band represents the in-plane vibration of the $\mathrm{sp}^{2}$ carbon atom; and the $\mathrm{D}$ band indicates carbon defects. ${ }^{62,63}$ The ratios of the intensity of the $\mathrm{D}$ band to that of the $\mathrm{G}$ band $\left(I_{\mathrm{d}} / I_{\mathrm{g}}\right)$ of $\mathrm{C}, \mathrm{C}-1, \mathrm{C}-1.5$ and C-2 were found to be $3.02,2.47,1.64$ and 2.26 , respectively. C1.5 with the lowest value of $I_{\mathrm{d}} / I_{\mathrm{g}}$ indicates that it has the fewest structural defects.

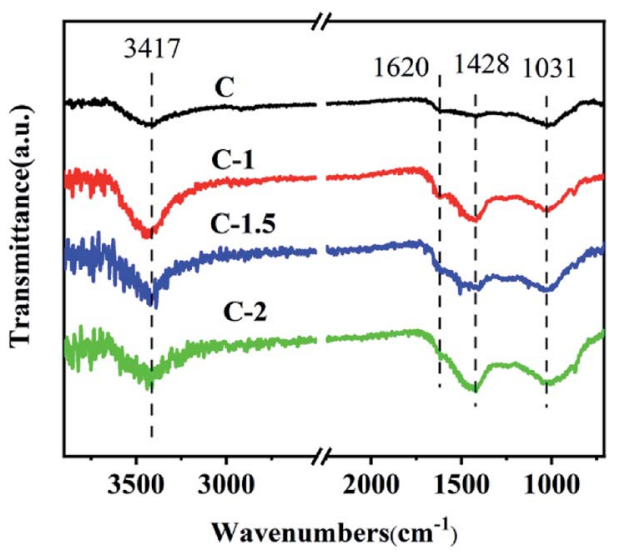

Fig. 3 FT-IR spectra of C, C-1, C-1.5 and C-2. 


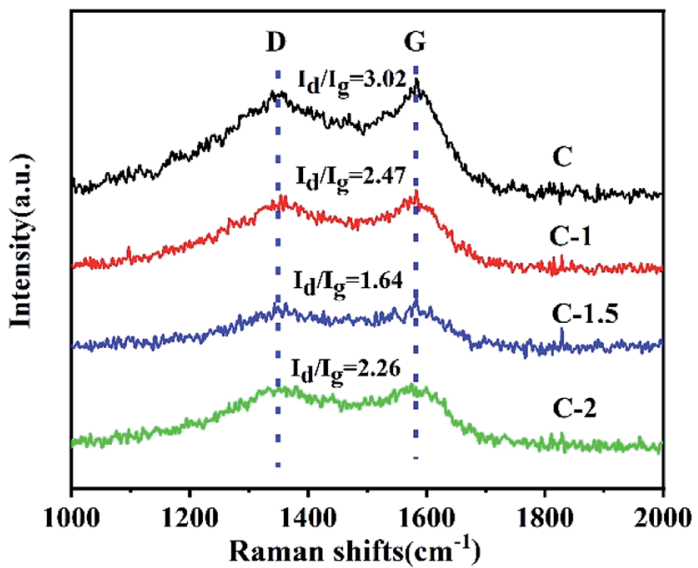

Fig. 4 Raman spectra of C, C-1, C-1.5 and C-2.

\subsection{Adsorption experiments}

Next, the adsorption performances of various carbon materials, including the carbon material prepared using the $\mathrm{NaHCO}_{3}$ assisted leavening method, were determined. The results are given in Fig. 5. C-1.5 removed the most sulfur from pentanethiol, achieving a relatively high adsorption ability. In contrast, when using the same experimental conditions, C showed a relatively poor adsorption ability, with only $23.8 \%$ of the sulfur having been removed. The percentages of sulfur removed from pentanethiol by the tested carbon materials followed the trend C-1.5 (99.7\%) > C-1 (98.6\%) > C-2 (79.9\%) > C (23.8\%). The excellent performances of the porous adsorbents may be ascribed to the Lewis acid-base derived from oxygen-containing functional groups in the porous carbon materials, strong van der Waals interactions, hierarchically porous structure and high specific surface areas.

Based on the above structural characterization and experimental results, a mechanism for the adsorption was derived. The strong Lewis acid $\mathrm{S}$ atoms in a pentanethiol molecule

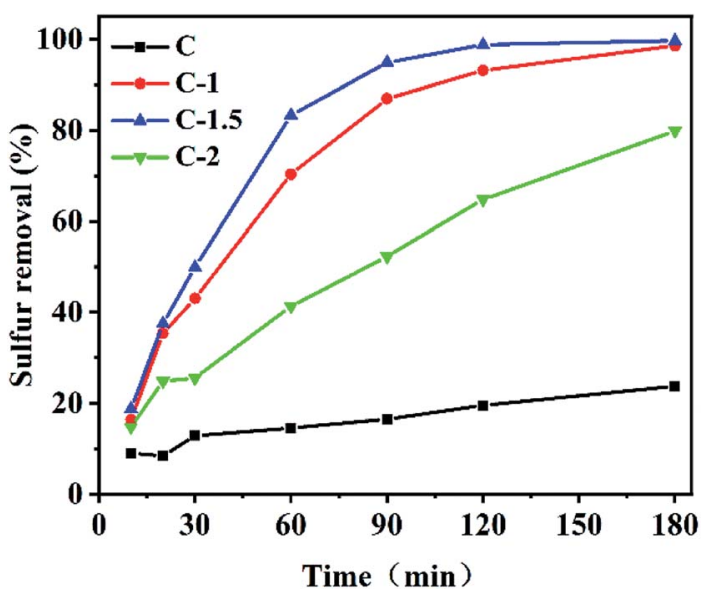

Fig. 5 The percentages of sulfur removed from pentanethiol by various carbon materials. Experimental conditions: $28 \mathrm{ppm}$ initial sulfur concentration, $T=25{ }^{\circ} \mathrm{C}, V$ (fuel) $=20 \mathrm{~mL}, m$ (adsorbent) $=0.1 \mathrm{~g}$, shaker agitation speed of $150 \mathrm{rpm}$ and atmospheric pressure.

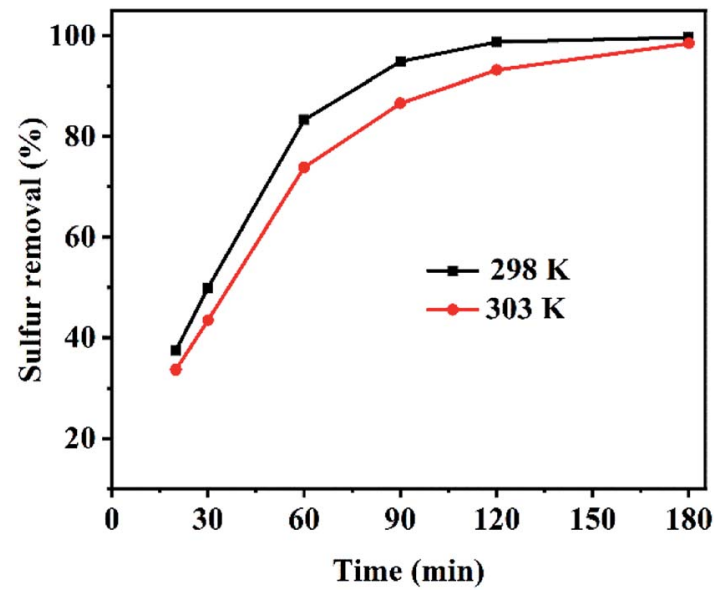

Fig. 6 Effect of temperature on the adsorption of pentanethiol by C1.5. Experimental conditions: $28 \mathrm{ppm}$ initial sulfur concentration, $V$ (fuel) $=20 \mathrm{~mL}, m$ (adsorbent) $=0.1 \mathrm{~g}$, shaker agitation speed of $150 \mathrm{rpm}$ and atmospheric pressure.

interacted with a pair of electrons from oxygen atoms in the carbon materials. ${ }^{31}$ The presence of macropores and mesopores contributed to the increased specific surface area of the carbon material, exposed more interior atoms, and facilitated mass transfer so that the adsorbents could easily contact the sulfides.

C-1.5 also removed a very high percentage (98.5\%) of sulfur at $303 \mathrm{~K}$, but slightly less so than the $99.7 \%$ removal at $298 \mathrm{~K}$ (Fig. 6). This small difference can be attributed to the exothermic nature of the adsorption process ${ }^{64-67}$

Concerning the industrial application prospects of C-1.5, the influence of the initial concentration of sulfur on the adsorption performance was considered. ${ }^{68}$ As shown in Fig. 7, C-1.5 displayed equilibrium adsorption capacities of 3.0, 5.6, 7.6, and $9.6 \mathrm{mg} \mathrm{S}$ per $\mathrm{g}$ adsorbent for the model fuels containing initial sulfur concentrations of $15,28,38$, and $48 \mathrm{ppm}$, respectively.

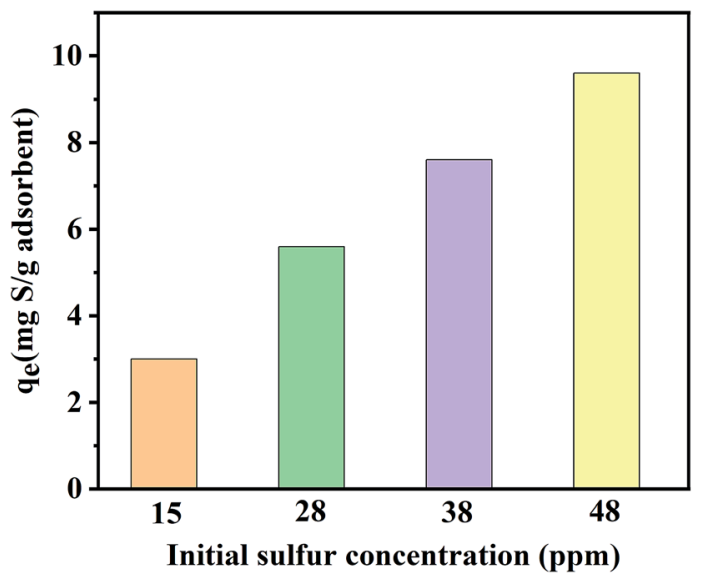

Fig. 7 Effect of the initial sulfur concentration on the adsorption of pentanethiol by $\mathrm{C}-1.5$. Experimental conditions: $T=25^{\circ} \mathrm{C}, V$ (fuel) $=$ $20 \mathrm{~mL}, m$ (adsorbent) $=0.1 \mathrm{~g}$, shaker agitation speed of $150 \mathrm{rpm}$, atmospheric pressure and contact time of 180 minutes. 


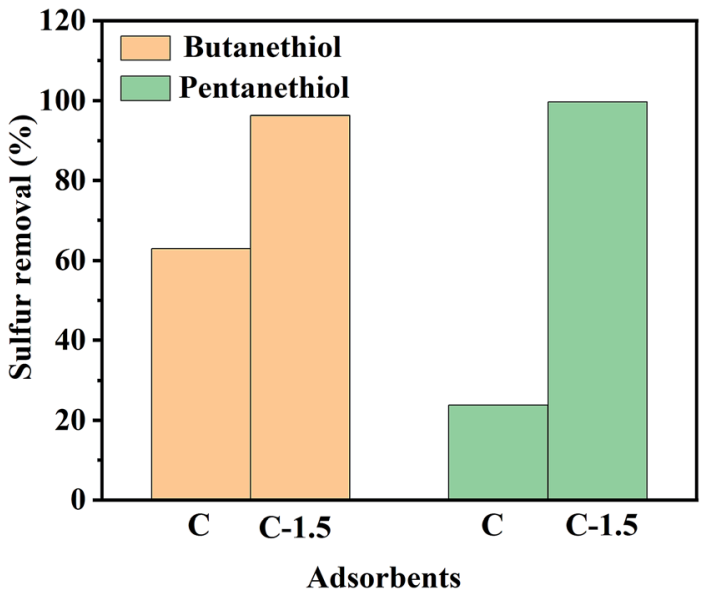

Fig. 8 The sulfur removal (\%) of C and C-1.5 for pentanethiol and butanethiol. Experimental conditions: 28 ppm initial sulfur concentration of pentanethiol, $27 \mathrm{ppm}$ initial sulfur concentration of butanethiol, $T=25^{\circ} \mathrm{C}, V$ (fuel) $=20 \mathrm{~mL}, m$ (adsorbent) $=0.1 \mathrm{~g}$, shaker agitation speed of $150 \mathrm{rpm}$, atmospheric pressure and contact time of $180 \mathrm{~min}$

It is worth noting that the sulfur removal of the adsorbent for this concentration range was above 97\%. This set of results indicates an obvious industrial application value of this material for the removal of low concentrations of sulfide.

To further explore the adsorption ability of the carbon materials for different thiols, butanethiol as well as pentanethiol were used as adsorbates, and the adsorption results are shown in Fig. 8. Most significantly, when $\mathrm{C}$ was used as the adsorbent, the percentages of sulfur removed from pentanethiol and butanethiol were $23.8 \%$ and $63 \%$, respectively, values much lower than the respective $99.7 \%$ and $96.3 \%$ values when C-1.5 was used as the adsorbent. As expected, the carbon material synthesized by the leavening method exhibited efficient adsorption.

\section{Conclusions}

We have developed a "leavening" method to treat used tires. This method was used to turn waste tires into a high-addedvalue product. The process was found to be efficient, mild, and facile, and may be generally applied to prepare porous carbon materials from other carbon sources. Benefiting from the randomly opened macropores, high specific surface area and abundant oxygen-containing functional groups, the resulting carbon materials exhibited an outstanding adsorptive desulfurization ability, showing a removal of $99.7 \%$ of sulfur from pentanethiol, and achieving such a nearly complete desulfurization using mild conditions. The outstanding performance was attributed to macropores and mesopores facilitating mass transfer and the strong Lewis acid $\mathrm{S}$ atoms in the pentanethiol molecules interacting with pairs of electrons from oxygen atoms in the carbon materials. Hence, this study may provide the impetus to prepare highly efficient adsorbents for the industrial production of clean fuels.

\section{Conflicts of interest}

There are no conflicts to declare.

\section{Acknowledgements}

This work was financially supported by the National Key R\&D Program of China (No. 2017YFB0306504), the National Natural Science Foundation of China (No. 21722604, 21606113, 21576122, 21878133).

\section{Notes and references}

1 K. X. Lee, G. Tsilomelekis and J. A. Valla, Appl. Catal., B, 2018, 234, 130-142.

2 X. Ren, Z. Liu, L. Dong, G. Miao, N. Liao, Z. Li and J. Xiao, AIChE J., 2018, 64, 2146-2159.

3 L. Sun, T. Su, J. Xu, D. Hao, W. Liao, Y. Zhao, W. Ren, C. Deng and H. Lu, Green Chem., 2019, 21, 2629-2634.

4 P. Wu, Y. Wu, L. Chen, J. He, M. Hua, F. Zhu, X. Chu, J. Xiong, M. He, W. Zhu and H. Li, Chem. Eng. J., 2020, 380, 122526.

5 W. Zhu, C. Wang, H. Li, P. Wu, S. Xun, W. Jiang, Z. Chen, Z. Zhao and H. Li, Green Chem., 2015, 17, 2464-2472.

6 L. Li, Y. Lu, H. Meng and C. Li, Fuel, 2019, 253, 802-810.

7 R. Ghubayra, C. Nuttall, S. Hodgkiss, M. Craven, E. F. Kozhevnikova and I. V. Kozhevnikov, Appl. Catal., B, 2019, 253, 309-316.

8 W. Zhou, Q. Wei, Y. Zhou, M. Liu, S. Ding and Q. Yang, Appl. Catal., B, 2018, 238, 212-224.

9 R. V. Mom, J. N. Louwen, J. W. M. Frenken and I. M. N. Groot, Nat. Commun., 2019, 10, 2546.

10 Y. Sun and R. Prins, Angew. Chem., Int. Ed., 2008, 47, 84788481.

11 H. Li, W. Zhu, S. Zhu, J. Xia, Y. Chang, W. Jiang, M. Zhang, Y. Zhou and H. Li, AIChE J., 2016, 62, 2087-2100.

12 T. Klimova, M. Calderon and J. Ramirez, Appl. Catal., A, 2003, 240, 29-40.

13 S. A. AL-Hammadi, A. M. Al-Amer and T. A. Saleh, Chem. Eng. J., 2018, 345, 242-251.

14 T. A. Saleh, S. A. AL-Hammadi, I. M. Abdullahi and M. Mustaqeem, J. Mol. Liq., 2018, 272, 715-721.

15 B. R. Fox, B. L. Brinich, J. L. Male, R. L. Hubbard, M. N. Siddiqui, T. A. Saleh and D. R. Tyler, Fuel, 2015, 156, 142-147.

16 X. Li, M. Hou, F. Li and H. Chua, Ind. Eng. Chem. Res., 2006, 45, 487-494.

17 M. H. Habibi, S. Tangestaninejad and B. Yadollahi, Appl. Catal., B, 2001, 33, 57-63.

18 R. Zavoianu, A. Cruceanu, O. D. Pavel, E. Angelescu, A. P. S. Dias and R. Birjega, React. Kinet., Mech. Catal., 2012, 105, 145-162.

19 D. W. Scott, D. L. Myers, H. Hill and O. Omadoko, Fuel, 2019, 242, 573-579.

20 J. He, J. Zhao and Y. Lan, J. Fuel Chem. Technol., 2009, 37, 485-488. 
21 S. S. Meshkat, O. Tavakoli, A. Rashidi and M. D. Esrafili, Ecotoxicol. Environ. Saf., 2018, 165, 533-539.

22 P. Tan, D. Xue, J. Zhu, Y. Jiang, Q. He, Z. Hou, X. Liu and L. Sun, AIChE J., 2018, 64, 3786-3793.

23 P. Tan, Y. Jiang, L. Sun, X. Liu, K. AlBahily, U. Ravon and A. Vinu, J. Mater. Chem. A, 2018, 6, 23978-24012.

24 J. Xiong, H. Li, L. Yang, J. Luo, Y. Chao, J. Pang and W. Zhu, AIChE J., 2017, 63, 3463-3469.

25 F. Seyedeyn-Azad, A. H. Ghandy, S. F. Aghamiri and R. Khaleghian-Moghadam, Fuel Process. Technol., 2009, 90, 1459-1463.

26 R. Barzamini, C. Falamaki and R. Mahmoudi, Fuel, 2014, 130, 46-53.

27 S. Bashkova, A. Bagreev and T. J. Bandosz, Environ. Sci. Technol., 2002, 36, 2777-2782.

28 H. Tamai, H. Nagoya and T. Shiono, J. Colloid Interface Sci., 2006, 300, 814-817.

29 S. W. Lee, W. M. A. W. Daud and M. G. Lee, J. Ind. Eng. Chem., 2010, 16, 973-977.

30 D. J. Kim and J. E. Yie, J. Colloid Interface Sci., 2005, 283, 311315.

31 S. S. Meshkat, A. Rashidi and O. Tavakoli, J. Nat. Gas Sci. Eng., 2018, 55, 288-297.

32 Z. Moghadaszadeh, M. R. Toosi and M. R. Zardoost, J. Mol. Model., 2019, 25, 138.

33 Y. Shi, X. Zhang and G. Liu, ACS Sustainable Chem. Eng., 2015, 3, 2237-2246.

34 Y. Shi, G. Liu, L. Wang and X. Zhang, Chem. Eng. J., 2015, 259, 771-778.

35 J. H. Kim, X. Ma, A. Zhou and C. Song, Catal. Today, 2006, 111, 74-83.

36 X. Li, H. Zhu, C. Liu, P. Yuan, Z. Lin, J. Yang, Y. Yue, Z. Bai, T. Wang and X. Bao, Ind. Eng. Chem. Res., 2018, 57, 1502015030.

37 G. I. Danmaliki and T. A. Saleh, Chem. Eng. J., 2017, 307, 914927.

38 T. A. Saleh and G. I. Danmaliki, J. Taiwan Inst. Chem. Eng., 2016, 60, 460-468.

39 G. I. Danmaliki, T. A. Saleh and A. A. Shamsuddeen, Chem. Eng. J., 2017, 313, 993-1003.

40 T. A. Saleh and G. I. Danmaliki, Process Saf. Environ. Prot., 2016, 102, 9-19.

41 T. A. Saleh, K. O. Sulaiman, S. A. Al-Hammadi, H. Dafalla and G. I. Danmaliki, J. Cleaner Prod., 2017, 154, 401-412.

42 T. A. Saleh, S. A. Al-Hammadi, A. Tanimu and K. Alhooshani, J. Colloid Interface Sci., 2018, 513, 779-787.

43 G. I. Danmaliki and T. A. Saleh, J. Cleaner Prod., 2016, 117, 50-55.

44 M. Hofman and R. Pietrzak, Chem. Eng. J., 2011, 170, 202208.
45 M. Tang, J. Deng, M. Li, X. Li, H. Li, Z. Chen and Y. Wang, Green Chem., 2016, 18, 6082-6090.

46 J. Deng, T. Xiong, F. Xu, M. Li, C. Han, Y. Gong, H. Wang and Y. Wang, Green Chem., 2015, 17, 4053-4060.

47 C. Wang, Z. Chen, X. Yao, Y. Chao, S. Xun, J. Xiong, L. Fan, W. Zhu and H. Li, Fuel, 2018, 230, 104-112.

48 T. A. Saleh, Environ. Sci. Pollut. Res., 2015, 22, 16721-16731. 49 T. A. Saleh, J. Water Supply: Res. Technol.-AQUA, 2015, 64, 892-903.

50 T. A. Saleh, Desalin. Water Treat., 2016, 57, 10730-10744.

51 J. Xiong, J. Luo, L. Yang, J. Pang, W. Zhu and H. Li, J. Ind. Eng. Chem., 2018, 64, 383-389.

52 X. Chen, M. Zhang, Y. Wei, H. Li, J. Liu, Q. Zhang, W. Zhu and H. Li, Inorg. Chem. Front., 2018, 5, 2478-2485.

53 S. Xun, W. Jiang, T. Guo, M. He, R. Ma, M. Zhang, W. Zhu and $\mathrm{H}$. Li, J. Colloid Interface Sci., 2019, 534, 239-247.

54 L. Lu, J. He, P. Wu, Y. Wu, Y. Chao, H. Li, D. Tao, L. Fan, H. Li and W. Zhu, Green Chem., 2018, 20, 4453-4460.

55 L. Fan, L. Yang, X. Y. Ni, J. Han, R. Guo and C. F. Zhang, Carbon, 2016, 107, 629-637.

56 T. A. Saleh, Appl. Surf. Sci., 2011, 257, 7746-7751.

57 T. A. Saleh, J. Cleaner Prod., 2018, 172, 2123-2132.

58 T. A. Saleh, S. A. AL-Hammadi and A. M. Al-Amer, Process Saf. Environ. Prot., 2019, 121, 165-174.

59 J. Romanos, M. Beckner, T. Rash, L. Firlej, B. Kuchta, P. Yu, G. Suppes, C. Wexler and P. Pfeifer, Nanotechnology, 2012, 23, 015401.

60 C. Troca-Torrado, M. Alexandre-Franco, C. FernandezGonzalez, M. Alfaro-Dominguez and V. Gomez-Serrano, Fuel Process. Technol., 2011, 92, 206-212.

61 C. Yang, H. Noguchi, K. Murata, M. Yudasaka, A. Hashimoto, S. Iijima and K. Kaneko, Adv. Mater., 2005, 17, 866-870.

62 M. Wang, Y. Lai, L. Fang, J. Li, F. Qin, K. Zhang and H. Lu, Int. J. Hydrogen Energy, 2015, 40, 16230-16237.

63 F. Pan, Z. Cao, Q. Zhao, H. Liang and J. Zhang, J. Power Sources, 2014, 272, 8-15.

64 J. Xiong, L. Yang, Y. H. Chao, J. Y. Pang, M. Zhang, W. S. Zhu and H. M. Li, ACS Sustainable Chem. Eng., 2016, 4, 44574464.

65 J. Xiong, L. Yang, Y. Chao, J. Pang, P. Wu, M. Zhang, W. Zhu and H. Li, Green Chem., 2016, 18, 3040-3047.

66 J. Xiong, W. Zhu, H. Li, L. Yang, Y. Chao, P. Wu, S. Xun, W. Jiang, M. Zhang and H. Li, J. Mater. Chem. A, 2015, 3, 12738-12747.

67 J. Luo, Y. Chao, Z. Tang, M. Hua, X. Li, Y. Wei, H. Ji, J. Xiong, W. Zhu and H. Li, Ind. Eng. Chem. Res., 2019, 58, 1330313312.

68 J. Xiong, W. Zhu, H. Li, W. Ding, Y. Chao, P. Wu, S. Xun, M. Zhang and H. Li, Green Chem., 2015, 17, 1647-1656. 